\title{
A Two-Amino Acid Difference in the Coat Protein of Satellite panicum mosaic virus Isolates Is Responsible for Differential Synergistic Interactions with Panicum mosaic virus
}

\author{
R. V. Chowda-Reddy, ${ }^{1}$ Nathan Palmer, ${ }^{1}$ Serge Edme, ${ }^{2}$ Gautam Sarath, ${ }^{2}$ Frank Kovacs, ${ }^{3}$ Gary Yuen, ${ }^{4}$ \\ Robert Mitchell, ${ }^{2}$ and Satyanarayana Tatineni ${ }^{1,4,+}$ \\ ${ }^{1}$ United States Department of Agriculture (USDA)-Agricultural Research Service (ARS), University of Nebraska-Lincoln, Lincoln, \\ NE 68583, U.S.A.; ${ }^{2}$ USDA-ARS and Department of Agronomy and Horticulture, University of Nebraska-Lincoln; ${ }^{3}$ Department of \\ Chemistry, University of Nebraska-Kearney, Kearney, NE 68849, U.S.A.; and ${ }^{4}$ Department of Plant Pathology, University of \\ Nebraska-Lincoln
}

Accepted 29 October 2018.

\begin{abstract}
Panicum mosaic virus (PMV) (genus Panicovirus, family Tombusviridae) and its molecular parasite, Satellite panicum mosaic virus (SPMV), synergistically interact in coinfected proso and pearl millet (Panicum miliaceum $\mathbf{L}$.) plants resulting in a severe symptom phenotype. In this study, we examined synergistic interactions between the isolates of PMV and SPMV by using PMV-NE, PMV85, SPMV-KS, and SPMV-Type as interacting partner viruses in different combinations. Coinfection of proso millet plants by PMV-NE and SPMV-KS elicited severe mosaic, chlorosis, stunting, and eventual plant death compared with moderate mosaic, chlorotic streaks, and stunting by PMV85 and SPMV-Type. In reciprocal combinations, coinfection of proso millet by either isolate of PMV with SPMV-KS but not with SPMV-Type elicited severe disease synergism, suggesting that SPMV-KS was the main contributor for efficient synergistic interaction with PMV isolates. Coinfection of proso millet plants by either isolate of PMV and SPMV-KS or SPMV-Type caused increased accumulation of coat protein $(C P)$ and genomic RNA copies of PMV, compared with infections by individual PMV isolates. Additionally, CP and genomic RNA copies of SPMV-KS accumulated at substantially higher levels, compared with SMPV-Type in coinfected proso millet plants with either isolate of PMV. Hybrid viruses between SPMV-KS and SPMV-Type revealed that SPMV isolates harboring a CP fragment with four differing amino acids at positions 18, 35, 59,
\end{abstract}

The United States Department of Agriculture (USDA) is an equal opportunity provider and employer. Mention of trade names or commercial products in this publication is solely for the purpose of providing specific information and does not imply recommendation or endorsement by the USDA.

${ }^{\dagger}$ Corresponding author: S. Tatineni;

E-mail: Satyanarayana.tatineni@ ars.usda.gov

Funding: This research was funded by the U.S. Department of Energy through grant DE-SC0016108.

*The $\boldsymbol{e}$-Xtra logo stands for "electronic extra" and indicates that one supplementary figure is published online.

This article is in the public domain and not copyrightable. It may be freely reprinted with customary crediting of the source. The American Phytopathological Society, 2019. and 98 were responsible for differential synergistic interactions with PMV in proso millet plants. Mutation of amino acid residues at these positions in different combinations in SPMV-KS, similar to those as in SPMV-Type or vice-versa, revealed that A35 and R98 in SPMV-KS CP play critical roles in enhanced synergistic interactions with PMV isolates. Taken together, these data suggest that the two distinct amino acids at positions 35 and 98 in the CP of SPMV-KS and SPMV-Type are involved in the differential synergistic interactions with the helper viruses.

Panicum mosaic virus (PMV) is a pathogen of potential economic importance in cellulosic ethanol-based bioenergy switchgrass (Panicum virgatum L.) breeding plots (Stewart et al. 2015), and it was first reported in 1953 from the state of Kansas in the United States (Sill and Pickett 1957). Moreover, PMV is ubiquitous in turfgrasses in the Gulf Coast and midwestern United States (Batten and Scholthof 2004). PMV is the type member of the genus Panicovirus in the family Tombusviridae with a monopartite positive-sense single-stranded (ss) RNA genome of approximately 4,300 nucleotides (nts) encapsidated in approximately $30-\mathrm{nm}$ icosahedral virions (Turina et al. 1998). The PMV genome is uncapped at the 5' end and lacks polyadenylation in the $3^{\prime}$ end; however, de novo polyadenylation of genomic RNA has been reported (Pyle 2015). The PMV genomic RNA encodes six open reading frames (ORFs). The p48 and p112 proteins are replicationassociated proteins that directly translate from the genomic RNA, while the coat protein $(\mathrm{CP}), \mathrm{p} 8, \mathrm{p} 6.6$, and $\mathrm{p} 15$ proteins are expressed through a polycistronic subgenomic RNA. These four proteins are involved in cell-to-cell and long-distance movement of PMV (Batten et al. 2006; Turina et al. 2000). PMV is unique in that it acts as a helper virus to Satellite panicum mosaic virus (SPMV) and two satellite RNAs of 375 and $444 \mathrm{nt}$ (satS and satC, respectively) (Masuta et al. 1987; Pyle et al. 2017).

Synergistic interactions facilitate coinfection of the same cell by unrelated viruses, which would result in a severe symptom phenotype compared with infections by individual viruses (Mascia and Gallitelli 2016; Syller 2012). In general, synergistic interactions cause increased virus titer of one or all interacting viruses compared with infections by individual viruses (Mascia and Gallitelli 2016; Syller 2012). Coinfection 
of PMV and SPMV cause disease synergism and is most commonly associated with St. Augustinegrass decline disease in turfgrass (Scholthof 1999; Scholthof et al. 1999). However, these interactions are different from other reported synergistic interactions because the partner viruses are a helper virus and its molecular parasitic satellite virus but not two independently self-replicating viruses. Hence, the effect of PMV and SPMV in synergistic interactions can be evaluated based on symptoms elicited by PMV in the presence or absence of SPMV but not coinfections compared with infections by individual viruses. PMV causes mild mosaic and mottling symptoms on proso and pearl millet plants, while coinfection of these millets by PMV and SPMV elicits chlorosis and stunting with increased accumulation of PMV genomic RNA and its p8 and CP (Scholthof 1999), indicating that SPMV is involved in synergistic interactions with PMV. Additionally, synergistic interactions between PMV and SPMV in Brachypodium distachyon caused misregulation of host-defense gene expression and changes in mRNA splicing patterns (Mandadi and Scholthof 2012, 2015; Mandadi et al. 2015).

SPMV has a positive-sense ssRNA genome of approximately $820 \mathrm{nts}$ encapsidated in 16-nm icosahedral virus particles (Ban and McPherson 1995; Masuta et al. 1987). The SPMV genome is monocistronic in nature, with an ORF encoding for a multifunctional 17-kDa CP (Masuta et al. 1987). The SPMV genomic RNA contains 5' - and 3'-untranslated regions (UTR) of 87 and 264 nts, respectively, with cis-acting elements involved in replication and movement of SPMV in the presence of PMV (Omarov et al. 2005; Qiu and Scholthof 2000). In addition to virion assembly, the CP of SPMV is also involved in synergistic interactions with its helper virus (Qiu and Scholthof 2001a; Scholthof 1999). Though CP is not absolutely required for SPMV replication and movement (Qiu and Scholthof 2000), the absence of CP debilitated systemic accumulation of SPMV RNA (Omarov et al. 2005) and facilitated rapid accumulation of defective interfering RNAs (Qiu and Scholthof 2001b). SPMV CP is a pathogenicity determinant, because expression of SPMV CP in a nonhost through a heterologous virus elicited severe symptoms by interfering with suppression of gene silencing (Qiu and Scholthof 2004). The crystal structure of SPMV CP indicated that the CP is an eight-stranded 'jelly roll' $\beta$-barrel with the $\mathrm{N}$-terminal arginine-rich motif $(\mathrm{N}$-ARM) extending into the interior of the virus particles predicted to interact with the SPMV genomic RNA (Ban and McPherson 1995; Makino et al. 2006; Qi and Scholthof 2008).

Synergistic interactions between PMV and SPMV have been studied extensively, using viruses derived from infectious cDNA clones of PMV isolate 85 (PMV85) (Turina et al. 1998) and SPMV isolate Type (SPMV-Type) (Qiu and Scholthof 2000; Scholthof 1999). There is no information on synergistic interactions between isolates of PMV and SPMV. In this study, infectious cDNA clones for a Nebraska isolate of PMV (pPMV-

Table 1. Sequence comparison of Panicum mosaic virus (PMV) isolates at nucleotide (nts) and amino acid (aa) levels ${ }^{\mathrm{a}}$

\begin{tabular}{lrrrrr}
\hline & \multicolumn{2}{c}{$\begin{array}{c}\text { Number of } \\
\text { changes }\end{array}$} & & \multicolumn{2}{c}{$\begin{array}{c}\text { Sequence identity } \\
(\%)\end{array}$} \\
\cline { 2 - 3 } Isolate & nts & aa & & nts & aa \\
\hline p48 & 60 & 11 & & 96 & 97 \\
p112 & 34 & 6 & & 98 & 99 \\
p8 & 3 & 0 & & 98 & 100 \\
p6.6 & 6 & 0 & & 97 & 100 \\
p15 & 11 & 8 & & 97 & 94 \\
Coat protein & 27 & 2 & & 98 & 99 \\
\hline
\end{tabular}

${ }^{a}$ Genome sequences of PMV-NE (this study) and PMV85 (Turina et al. 1998) were used for sequence comparison.
NE) from switchgrass and a Kansas isolate of SPMV (pSPMVKS) were developed. Coinfection of proso millet (Panicum miliaceum L.) seedlings by PMV-NE and SPMV-KS elicited severe chlorosis, stunting, and eventual death of plants compared with moderate chlorotic streaks and stunting of plants by PMV85 and SPMV-Type. In reciprocal experiments, proso millet plants coinfected by PMV85 and SPMV-KS but not by PMV-NE and SPMV-Type elicited severe chlorosis and stunting symptoms, indicating that SPMV-KS facilitated efficient synergistic interactions with PMV isolates. Genome sequence exchanges between SPMV-KS and SPMV-Type and mutational analyses revealed that two amino acid residues at positions 35 and 98 in the CP ORFs of SPMV isolates are responsible for differential synergistic interactions with the helper virus.

\section{RESULTS}

\section{Genome sequence of PMV-NE.}

Total RNA was isolated from switchgrass rhizomes and flag leaves collected between 2010 and 2012 from the Eastern Nebraska Research and Extension Center (ENREC), University of Nebraska-Lincoln, near Mead, Nebraska, and was subjected to RNA-Seq. The PMV-specific sequences were identified by aligning RNA-Seq reads to a Kansas isolate of PMV (PMV85, GenBank accession number U55002). This alignment was used to generate a Nebraska PMV population consensus genome, and this consensus sequence was named PMV-NE. The PMVNE genome is 4,324 nts long, possessed $97 \%$ sequence identity with PMV85 (4,326 nts) at the nucleotide level. Compared with PMV85, the Nebraska isolate of PMV has 128 nucleotide changes and two nucleotide deletions (Table 1). The highest number of nucleotide variation was found in the p48 ORF, with 96\% identity at the nucleotide level. However, the most compelling diversity between PMV-NE and PMV85 at the amino acid level was found in the p15 ORF, with $94 \%$ identity. The p8 and p6.6 ORFs exhibited 2 to $3 \%$ synonymous changes, while the p112 and CP ORFs possess 98 and $99 \%$ identity at the nucleotide and amino acid level, respectively (Table 1). The SPMV sequence was not detected in the RNA-Seq datasets, perhaps due to the library preparation methodologies; hence, a Kansas isolate of SPMV (SPMV-KS, GenBank accession number M17182) was used in this investigation.

\section{Examining synergistic interactions between isolates of PMV and SPMV revealed that the SPMV-KS isolate elicited augmented disease synergism in proso millet.}

The full-length cDNA copies of PMV-NE and SPMV-KS were synthesized and ligated into pUC57-Mini at the EcoRV restriction site (GenScript, Piscataway, NJ, U.S.A.) to obtain pPMV-NE and pSPMV-KS, respectively. A unique XbaI restriction site followed by a T7 RNA polymerase promoter and a unique NotI restriction site were respectively introduced at the $5^{\prime}$ and $3^{\prime}$ termini of PMV and SPMV genomes (Fig. 1). In-vitro RNA transcripts generated from NotI-linearized pPMV-NE and pSPMV-KS were inoculated onto proso millet seedlings at the two-leaf stage, as described by Tatineni et al. (2011). As positive controls, in-vitro RNA transcripts generated from pPMV85 (Turina et al. 1998) and pSPMV-Type (Scholthof 1999; Qiu and Scholthof 2000) were also used to inoculate proso millet seedlings. Proso millet plants infected by PMV-NE or PMV85 elicited mild mottling symptoms with slight stunting of plants at 14 days postinoculation (dpi) (Fig. 2A and B). In contrast, proso millet plants coinfected by PMV-NE and SPMV-KS elicited severe systemic leaf chlorosis followed by purple yellowish color with severe stunting and death of the majority of plants between 14 and 28 dpi (Fig. 2A and B). However, coinfection of proso millet plants by PMV85 and 
SPMV-Type elicited moderate long chlorotic streaks with no severe chlorosis and moderate stunting of plants (Fig. 2A and B). Taken together, coinfection of proso millet plants by PMVNE and SPMV-KS elicited more severe symptoms compared with those by PMV85 and SPMV-Type, suggesting that PMVNE or SPMV-KS, or both, are responsible for severe disease synergism in proso millet plants.

Proso millet seedlings were next inoculated at the two-leaf stage with in-vitro transcripts of PMV85+SPMV-KS or PMVNE+SPMV-Type. Seedlings coinfected by PMV85+SPMV-KS elicited severe leaf chlorosis with purple yellowish color and severe stunting of plants, similar to that elicited by PMV-NE+ SPMV-KS. However, coinfection of proso millet plants by PMVNE+SPMV-Type elicited symptoms similar to those by PMV85+ SPMV-Type, i.e., with moderate chlorotic streaks and stunting of plants (Fig. 2A and B). Taken together, coinfection of proso millet plants with either isolate of PMV plus SPMV-KS but not with SPMV-Type elicited a substantially more-severe symptom phenotype, suggesting that SPMV-KS was responsible for the augmented disease synergism observed in proso millet plants.

PMV-NE

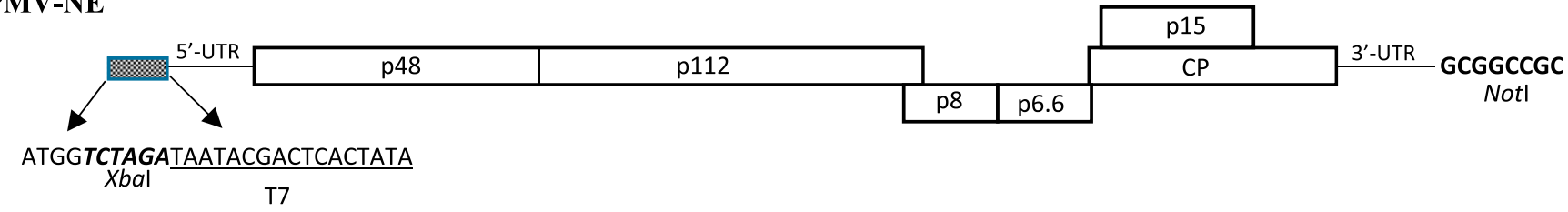

SPMV-KS

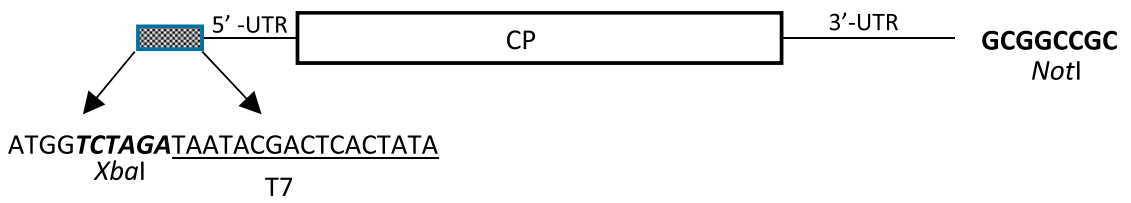

Fig. 1. Schematic representation of genome organization of Panicum mosaic virus Nebraska isolate (PMV-NE) and Satellite panicum mosaic virus Kansas isolate (SPMV-KS). Genomic organization of PMV-NE and its encoded open reading frames (ORFs) (open boxes) with 5'-and 3'-untranslated regions (UTR) are indicated. Genomic organization of SPMV-KS and its encoded ORF (CP [coat protein]). A unique XbaI restriction site (in bold italics), followed by T7 RNA polymerase promoter sequence (underlined) and a NotI restriction site introduced at the 5' and $3^{\prime}$ ends, respectively, of the genomes of PMV and SPMV are indicated.

A

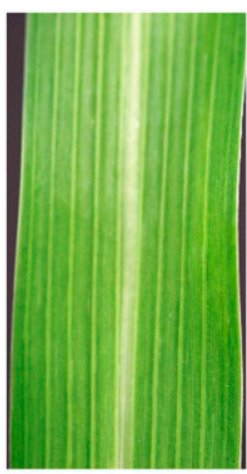

Mock

B

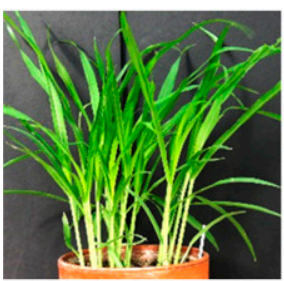

Mock

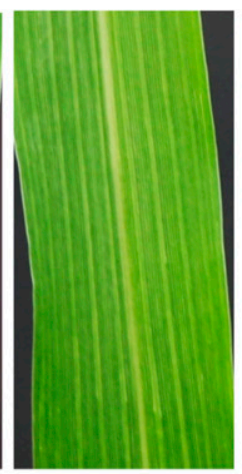

PMV85

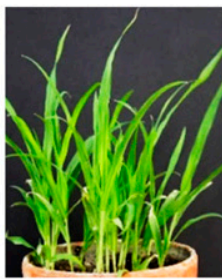

PMV85

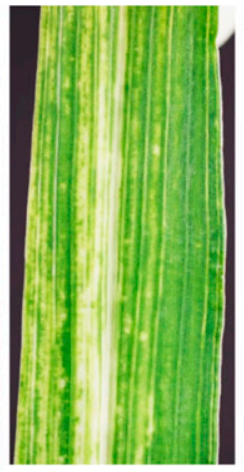

PMV85+SPMV-Type

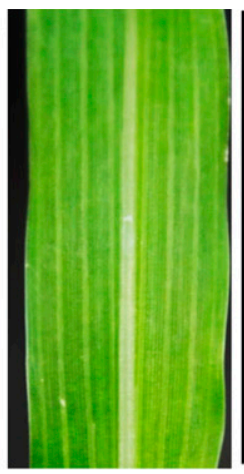

PMV-NE

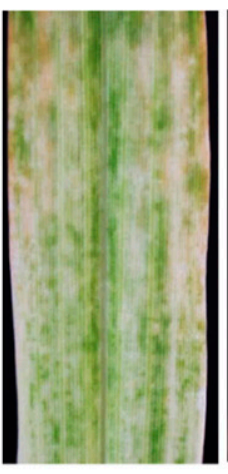

PMV-NE+SPMV-KS

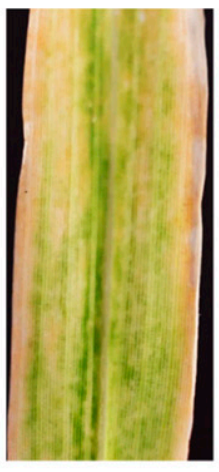

PMV85+SPMV-KS

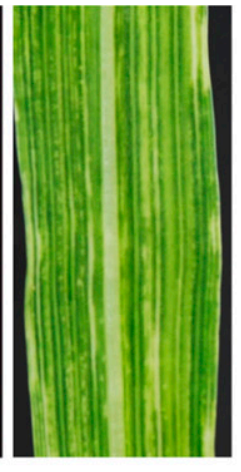

PMV-NE+SPMV-Type

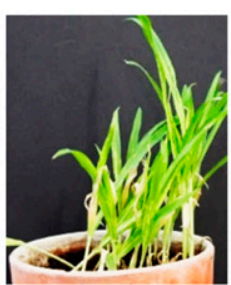

PMV85+SPMV-Type

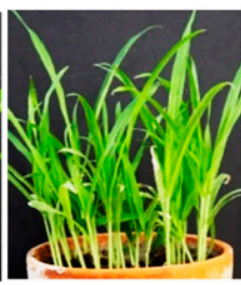

PMV-NE

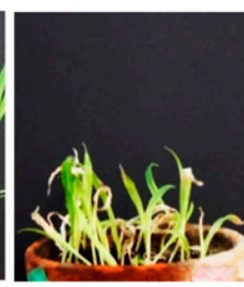

PMV-NE+SPMV-KS

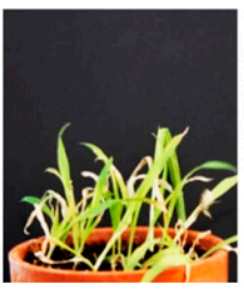

PMV85+SPMV-KS

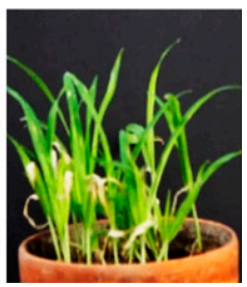

PMV-NE+SPMV-Type

Fig. 2. Differential synergistic interactions between Panicum mosaic virus (PMV-NE or PMV85) and Satellite panicum mosaic virus (SPMV-KS or SPMVType) isolates on proso millet. A, Symptoms elicited by PMV or PMV+SPMV on upper uninoculated leaves of proso millet plants at 14 days postinoculation (dpi). Proso millet seedlings were inoculated with in-vitro transcripts of PMV85, PMV85+SPMV-Type, PMV-NE, PMV-NE+SPMV-KS, PMV85+SPMV$\mathrm{KS}$, and PMV-NE+SPMV-Type at the two-leaf stage. B, A group of proso millet plants infected by PMV or PMV+SPMV isolates in different combinations at $14 \mathrm{dpi}$, showing the effect of coinfection on plant growth and symptom severity. Note that severe synergistic interactions were observed on proso millet plants coinfected by either isolate of PMV with SPMV-KS, with severe stunting and leaf chlorosis, compared with moderate stunting and leaf chlorosis with SPMV-Type. 
Coinfection of proso millet plants by PMV and SPMV caused increased accumulation of PMV and persistent accumulation of SPMV-KS compared with SPMV-Type.

The effect of differential synergistic interactions of SPMV isolates with PMV isolates on accumulation of genomic RNA copies of PMV and SPMV in proso millet plants at 14 and $21 \mathrm{dpi}$ was examined by reverse transcription real-time polymerase
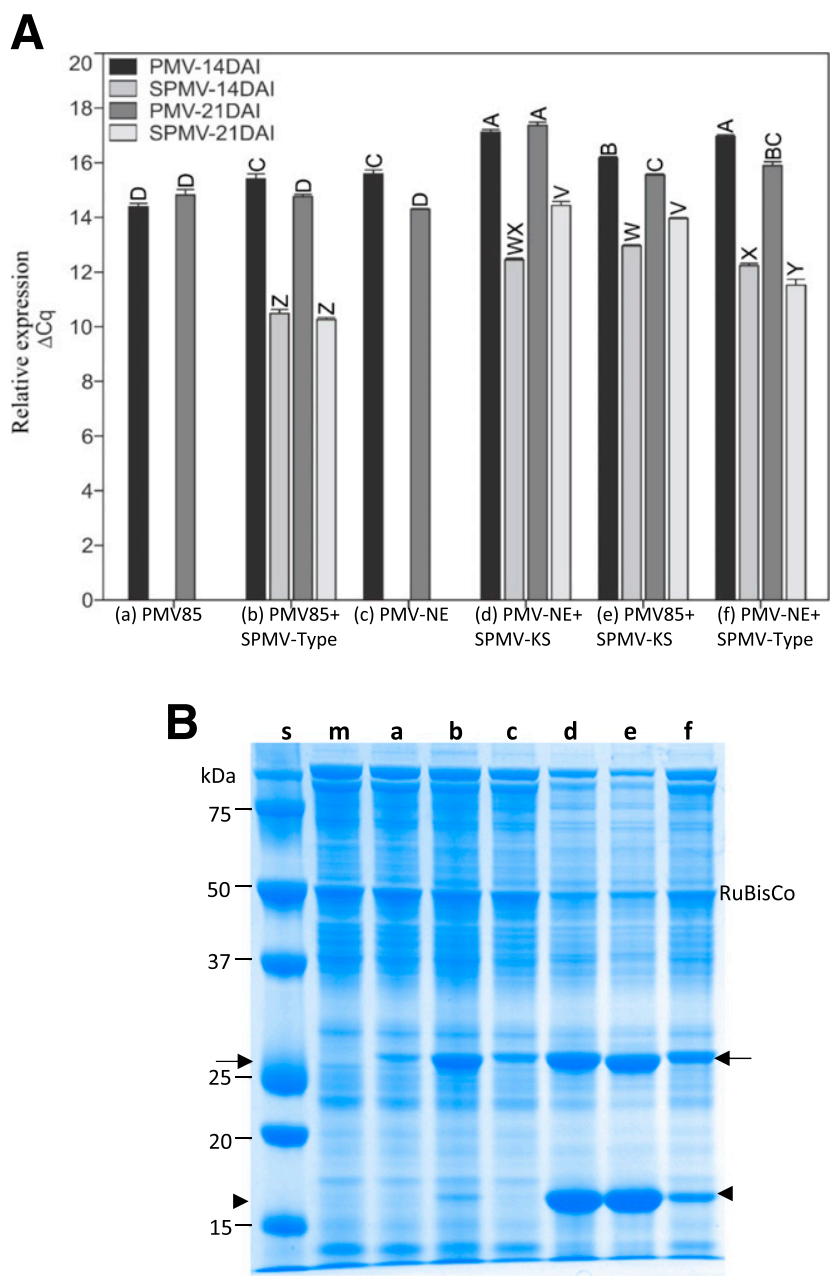

Fig. 3. Coinfection of proso millet plants by Panicum mosaic virus (PMV) and Satellite panicum mosaic virus (SPMV) caused enhanced accumulation of PMV genomic RNA copies and coat protein (CP). A, Reverse transcription real-time polymerase chain reaction analyses of PMV and SPMV genomic RNAs from proso millet plants. The PMV and SPMV genomic RNA copies were quantified from PMV- and PMV+SPMV-infected proso millet plants and PMV+SPMV-infected proso millet plants, respectively. The relative expression of genomic RNA copies of PMV and SPMV was determined at 14 and 21 days after inoculation (dai). Mean separations were calculated using Tukey's highly significant difference test in JMP 12 . Letters A, B, C, and D indicate the mean separations of relative expression of PMV genomic RNA at $P=0.05$, while letters $\mathrm{W}, \mathrm{X}, \mathrm{Y}$, and $\mathrm{Z}$ indicate mean separations of relative expression of SPMV genomic RNA at $P=$ 0.05. The same letters above individual bars indicate non-significance, whereas different letters indicate significance. Virus combinations that are infected proso millet seedlings are indicated on the $x$ axis. B, Sodium dodecyl sulfate-polyacrylamide gel electrophoresis analysis of total proteins (5 $\mu \mathrm{g}$ per lane) from PMV- or PMV+SPMV-infected proso millet leaves. The location of a $26-\mathrm{kDa}$ CP of PMV and a $17-\mathrm{kDa}$ CP of SPMV is indicated with arrows and arrow heads, respectively. The location of host RuBisCo protein is indicated at the right. Samples loaded in lanes ' $a$ ' through ' $\mathrm{f}$ ' are the same as in A. Lane ' $\mathrm{m}$ ' shows buffer-inoculated proso millet and lane s shows protein molecular weight standards. Note the increased accumulation of PMV in PMV+SPMV-infected proso millet compared with PMV-infected plants and enhanced accumulation of SPMVKS CP compared with SPMV-Type in coinfected proso millet plants. chain reaction (RT-qPCR). The RT-qPCR assay was performed on total RNA isolated from proso millet leaves singly infected by PMV-NE or PMV85 and doubly infected by either isolate of PMV (PMV-NE or PMV85) plus SPMV-KS or SPMV-Type at 14 and 21 dpi (Fig. 3A).

Compared with infection by PMV85 alone, coinfection of proso millet plants with PMV85+SPMV-Type caused statistically significant increase of PMV85 genomic RNA accumulation by $1.02 \log _{2}$ fold change at $14 \mathrm{dpi}$ but not at $21 \mathrm{dpi}$ (Fig. $3 \mathrm{~A})$. In contrast, compared with infection by PMV-NE alone, coinfection of proso millet plants by PMV-NE+SPMV-KS was more persistent, causing a statistically significant increase of 1.52 and $3.08 \log _{2}$ fold change of PMV-NE genomic RNA accumulation at 14 and $21 \mathrm{dpi}$, respectively (Fig. 3A). In reciprocal combinations, coinfection of proso millet plants by PMV85+SPMV-KS or PMV-NE+SPMV-Type caused a statistically significant increase in PMV genomic RNA accumulation by 1.78 and $1.36 \log _{2}$ fold change at $14 \mathrm{dpi}$, and 0.74 and $1.60 \log _{2}$ fold change at $21 \mathrm{dpi}$, respectively (Fig. 3A). Taken together, these data suggest that coinfection of proso millet plants by PMV+SPMV isolates resulted in significantly increased accumulation of PMV genomic RNA copies compared with infections by PMV isolates alone.

Accumulation of SPMV genomic RNA copies in coinfected proso millet plants at 14 and 21 dpi was compared between SMPV-KS and SPMV-Type isolates. Accumulation of SPMVKS in proso millet plants coinfected by SPMV-KS and PMVNE or PMV85 increased by 0.99 to $1.99 \log _{2}$ fold change at 21 dpi compared with 14 dpi (Fig. 3A). In contrast, accumulation of SPMV-Type was decreased by 0.22 to $0.71 \log _{2}$ fold change in the presence of PMV85 or PMV-NE at 21 dpi compared with 14 dpi (Fig. 3A). These data suggest that severe synergistic interactions by coinfecting proso millet plants with SPMV-KS plus either isolate of PMV caused persistent accumulation of SPMV genomic RNA copies.

Additionally, accumulation of CPs of PMV and SPMV was examined, in proso millet plants infected by PMV or PMV plus SPMV isolates at $14 \mathrm{dpi}$, by separating total proteins through sodium dodecyl sulfate-polyacrylamide gel electrophoresis (SDS-PAGE) followed by Coomassie Brilliant Blue R-250 staining (Fig. 3B). Accumulation of CPs of PMV85 and PMV$\mathrm{NE}$ was substantially increased in proso millet plants coinfected with either SPMV isolate compared with infections by PMV alone (Fig. 3B). In contrast, the CPs of SPMV-Type and SPMV-KS were differentially accumulated in proso millet plants coinfected with either isolate of PMV. The CP of SPMVKS was accumulated at substantially increased levels in proso millet plants coinfected with either PMV isolate as compared with extracts from plants coinfected with either PMV isolate and SPMV-Type (Fig. 3B). Taken together, these data suggest that increased symptom phenotype of proso millet plants coinfected by SPMV isolates with either isolate of PMV was due to increased accumulation of SPMV CP.

\section{Sequence difference between SPMV-KS and SPMV-Type isolates.}

To further investigate the SPMV determinants responsible for differential synergistic interactions with PMV, the cDNA clones of SPMV-KS and SPMV-Type were completely sequenced. SPMV-KS is $826 \mathrm{nts}$ long with differences at the 12-nt positions when compared with the 825-nt long SPMV-Type, and both isolates shared $98.5 \%$ homology at the nucleotide level (Supplementary Fig. S1). The CP ORF of SPMV isolates possessed $98 \%$ sequence homology at the nucleotide and amino acid levels, whereas the $3^{\prime}$-UTR showed $99 \%$ homology. Four amino acid differences (A18T, A35D, A59S, and R98M) were found between the CP of SPMV-KS and SPMV-Type isolates. 
The CP of SPMV is responsible for differential synergistic interaction with PMV isolates.

The genetic determinants of SPMV-KS responsible for the augmented synergistic interaction with PMV isolates was mapped by reciprocally exchanging the two fragments of the SPMV genome between SpeI (nt 63) and MscI (nt 457) (fragment 1) and $M s c I$ (nt 457) and the $3^{\prime}$ end (fragment 2) between SPMV-KS and SPMV-Type isolates (Fig. 4A). Fragments 1 and

A

a. SPMV-KS

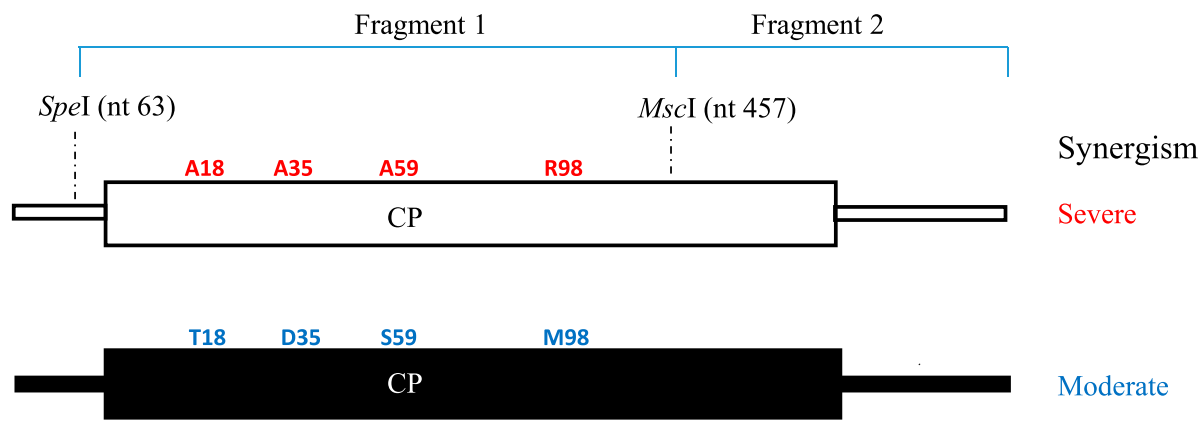

c. SPMV-KS_SPMV-Type CP

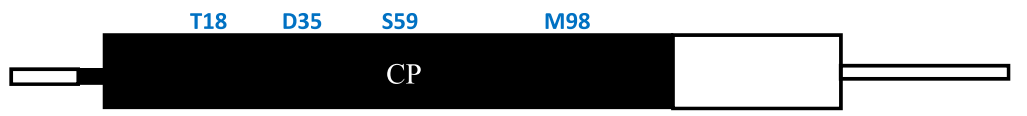

Moderate

d. SPMV-KS_SPMV-Type 3'-UTR

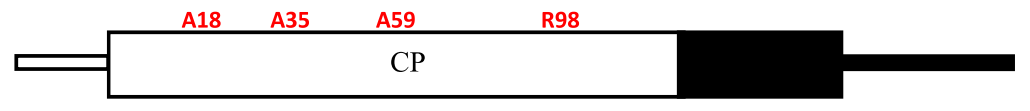

Severe

e. SPMV-Type_SPMV-KS CP

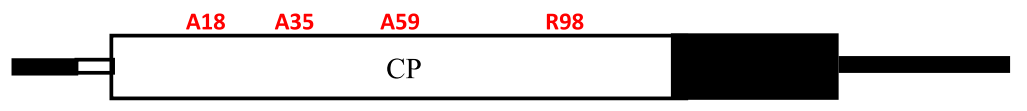

Severe

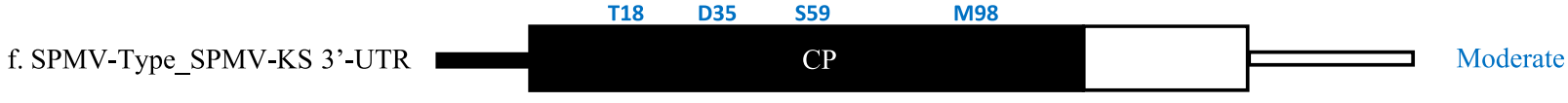

B
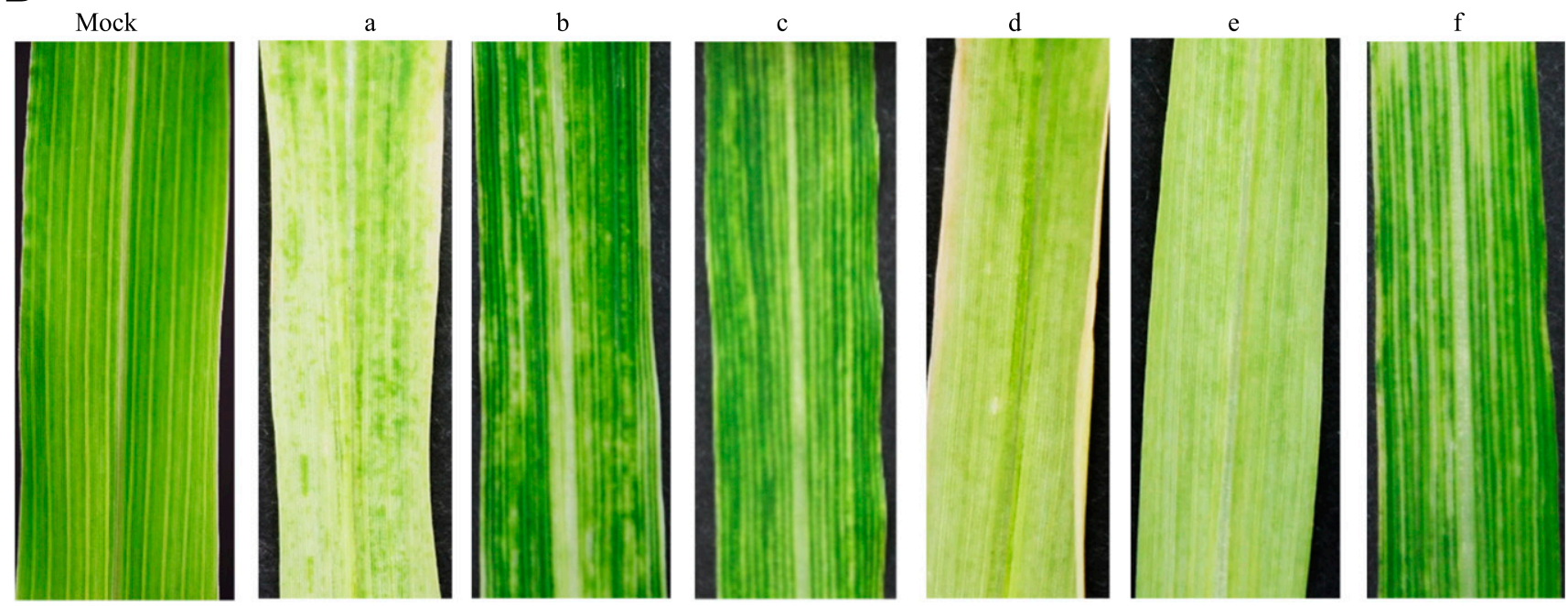

Fig. 4. Satellite panicum mosaic virus (SPMV) coat protein (CP) is responsible for differential synergistic interactions with Panicum mosaic virus (PMV) isolates. A, Schematic representation of genomic organization of SPMV hybrids generated between SPMV-KS (in white) and SPMV-Type (in black). SPMV hybrids created by exchanging genomic regions between nucleotides (nts) 63 and 457 (fragment 1) and nts 457 and the $3^{\prime}$ terminal region (fragment 2), using the indicated unique restriction enzyme sites. Amino acids that differ between the CPs of SPMV-KS and SPMV-Type are indicated. Synergistic interaction phenotype of PMV coinfection with wild type or hybrid SPMVs on proso millet plants are indicated at the right. B, Symptoms induced by PMV coinfection with wild-type SPMV isolates or SPMV hybrids on proso millet leaves at 14 days postinoculation. Mock, buffer inoculation; a, SPMV-KS; b, SPMV-Type; c, SPMV-KS_SPMV-Type CP; d, SPMV-KS_SPMV-Type 3' UTR (untranslated region); e, SPMV-Type_SPMV-KS CP; and f, SPMV-Type_SPMV-KS 3' UTR. Note that coinfection of proso millet plants by PMV plus SPMV hybrids harboring SPMV-KS CP sequence elicited enhanced disease synergism. 
2 of SPMV-KS were independently replaced with those from SPMV-Type to obtain PSPMV-KS_SPMV-Type CP and pSPMV-KS_SPMV-Type 3'-UTR, respectively (Fig. 4A, columns c and d). Similarly, fragments 1 and 2 from SPMV-Type were precisely replaced with those from SPMV-KS to obtain pSPMV-Type_SPMV-KS CP and pSPMV-Type_SPMV-KS 3'UTR, respectively (Fig. 4A, columns e and f).

In-vitro transcripts of these hybrid viruses were coinoculated independently with both helper viruses onto proso millet seedlings at the two-leaf stage. Proso millet plants coinfected with either isolates of PMV plus SPMV-KS_SPMV-Type 3'UTR or SPMV-Type_SPMV-KS CP elicited augmented disease synergism with severe leaf chlorosis and stunting of plants during the 14- to 21-dpi observation (Fig. 4B, columns d and e). In contrast, proso millet plants coinfected with PMV isolates plus SPMV-KS_SPMV-Type CP or SPMV-Type_SPMV-KS 3'UTR elicited moderate leaf chlorosis and stunting of plants during the 14- to 21-dpi period (Fig. 4B, columns $\mathrm{c}$ and $\mathrm{f}$ ). These data indicated that hybrid viruses with the $\mathrm{CP}$ from SPMV-KS but not from 3'-UTR induced enhanced synergistic

A

a. SPMV-KS

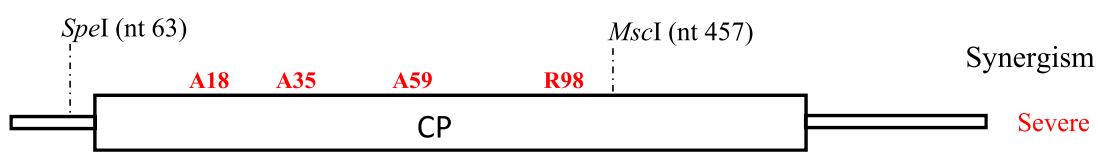

b. SPMV-Type

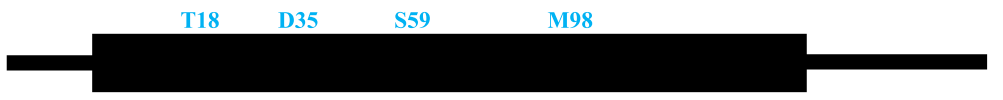

Moderate

c. SPMV-KS_TAAR

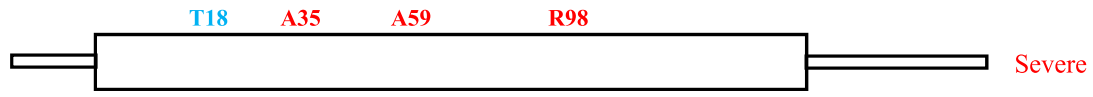

d. SPMV-KS_AASR

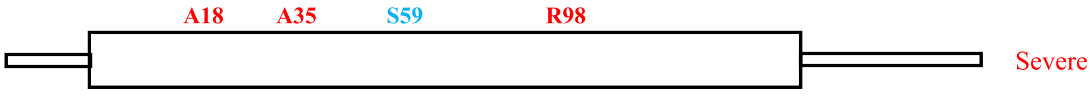

e. SPMV-KS_AAAM

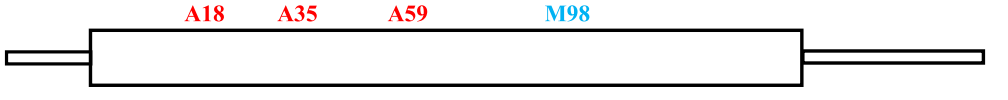

f. SPMV-KS ADSR

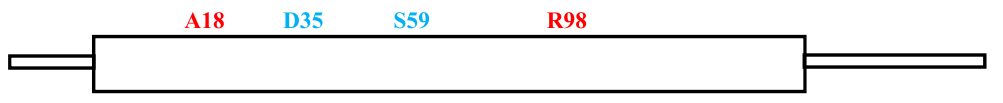

g. SPMV-KS_TDSR
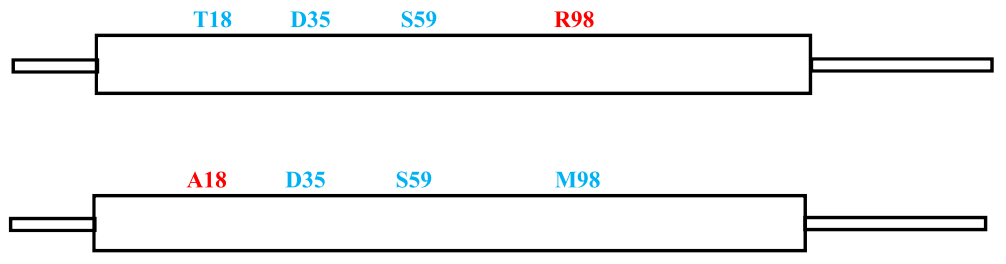

Moderate

B

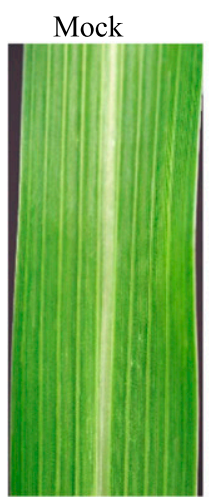

a

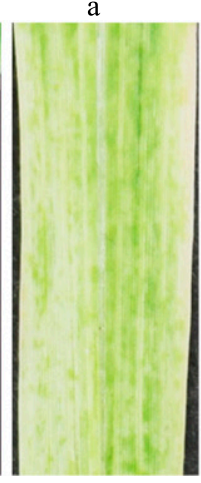

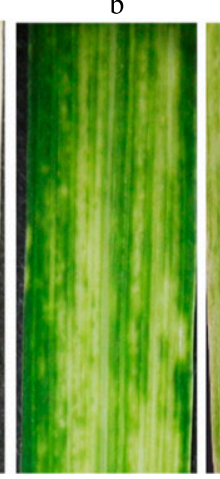

c

d

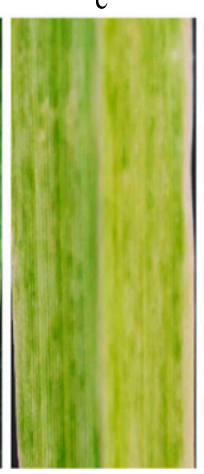

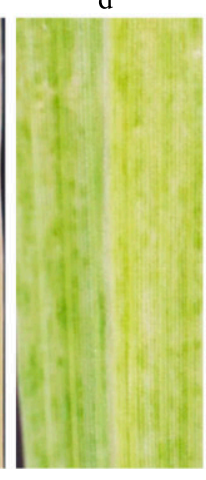

e

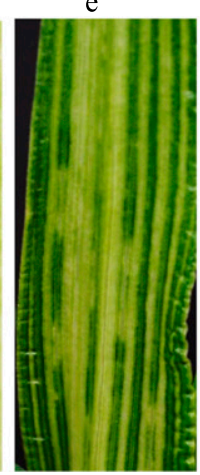

f

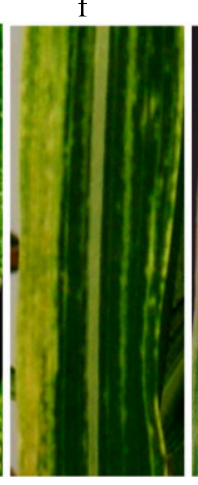

$\mathrm{g}$

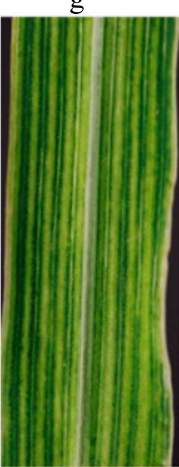

h

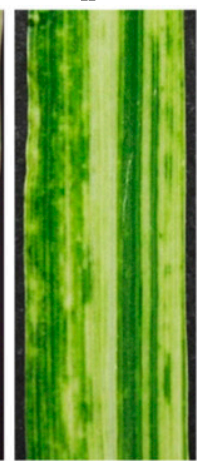

Fig. 5. Satellite panicum mosaic virus (SPMV) coat protein (CP) amino acid residues 35 and 98 are responsible for differential synergistic interaction with Panicum mosaic virus (PMV) isolates in proso millet plants. A, Genomic organization of SPMV-KS (a) and SPMV-Type (b), and single (c to e), double (f), and triple ( $\mathrm{g}$ and $\mathrm{h}$ ) amino acid CP mutants of SPMV-KS. SPMV-KS CP amino acids that differ from those of SPMV-Type were mutated singly (SPMV-KS_TAAR, SPMV-KS_AASR, and SPMV-KS_AASM), doubly (SPMV-KS_ADSR), and triply (SPMV-KS_TDSR and SPMV-KS_ADSM) to those as in SPMV-Type. The level of disease synergism elicited in coinfected proso millet plants by PMV and SPMV-KS mutants is indicated on the right. B, Symptoms elicited by SPMV mutants in coinfected proso millet plants with PMV-NE at 14 days postinoculation (a to $\mathrm{h}$ ). Mock is buffer-inoculated proso millet. Note that coinfection of SPMV-KS with mutation of amino acids at positions 18 or 59 elicited severe disease synergism while mutation of amino acid 98 induced moderate synergism. SPMV-KS with double mutation of amino acids 35 and 59 and triple mutation of amino acids at 18, 35, and 59 or 35, 59, and 98 induced moderate disease synergism with PMV. 
interaction with the helper virus. Moreover, a synonymous change at the $3^{\prime}$ end of the $\mathrm{CP}$ and three nucleotide changes found in the $3^{\prime}$-UTR in fragment 2 did not play a significant role in differential synergistic interactions with helper viruses. Collectively, these data suggest that CPs of SPMV isolates most likely played a crucial role in differential synergistic interactions with PMV isolates in doubly infected proso millet plants. Since each SPMV isolate similarly interacted with either isolate of PMV in the above experiments, PMV-NE isolate was used in all subsequent synergistic interactions with SPMV mutants.

\section{Amino acids A35 and R98 in the CP ORF of SPMV-KS} are responsible for enhanced synergistic interactions with PMV isolates in proso millet.

The effects of the SPMV-KS CP amino acids A18, A35, A59, and R98, which differ from those of SPMV-Type, were examined, for enhanced synergistic interactions with PMV isolates, by replacing these amino acids in different combinations with those of SPMV-Type (Fig. 5A). Amino acids A18, A59, and R98 were mutated individually to T18, S59, and M98 in mutants SPMV-KS_TAAR, SPMV-KS_AASR, and SPMVKS_AAAM, respectively (Fig. 5A). We next engineered a double mutant by changing amino acids A35 and A59 to D35 and S59 in SPMV-KS_ADSR. Finally, triple mutants were created by changing A18, A35, and A59 and A35, A59, and R98 to T18, D35, and S59 and D35, S59, and M98 in SPMVKS_TDSR and SPMV-KS_ADSM, respectively (Fig. 5A).

In-vitro transcripts of SPMV-KS CP mutants were coinoculated with PMV-NE onto proso millet seedlings at the two-leaf stage. Proso millet plants infected by SPMV-KS_TAAR or SPMV-KS_AASR elicited severe synergistic interactions similar to wild-type SPMV-KS (Fig. 5B, columns c and d compared

A

Synergism

a. SPMV-Type

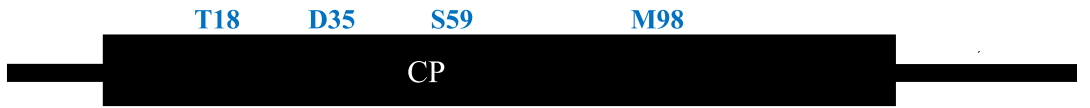

Moderate

b. SPMV-KS

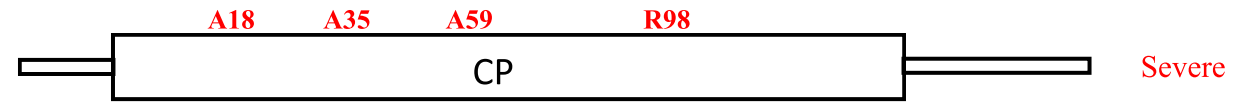

c. SPMV-Type_D35A

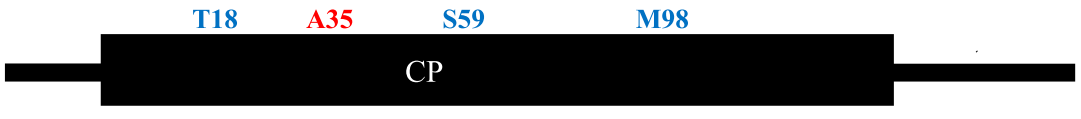

Moderately severe

d. SPMV-Type_M98R

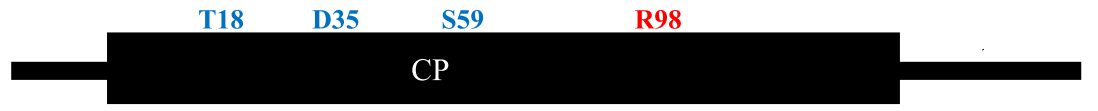

Severe

e. SPMV-Type_D35A+M98+R

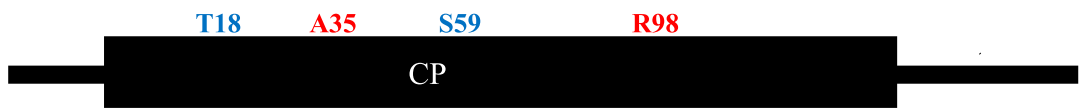

Severe

B
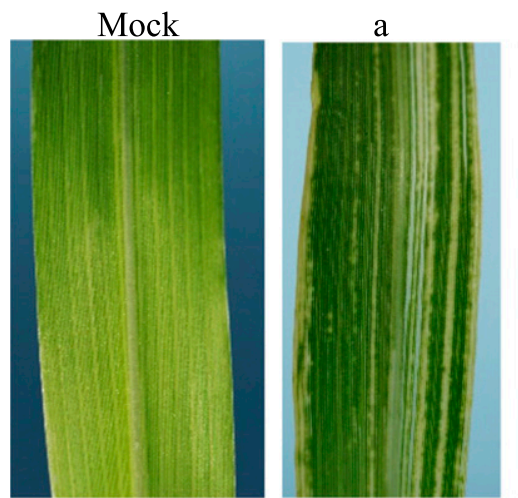

$\mathrm{b}$
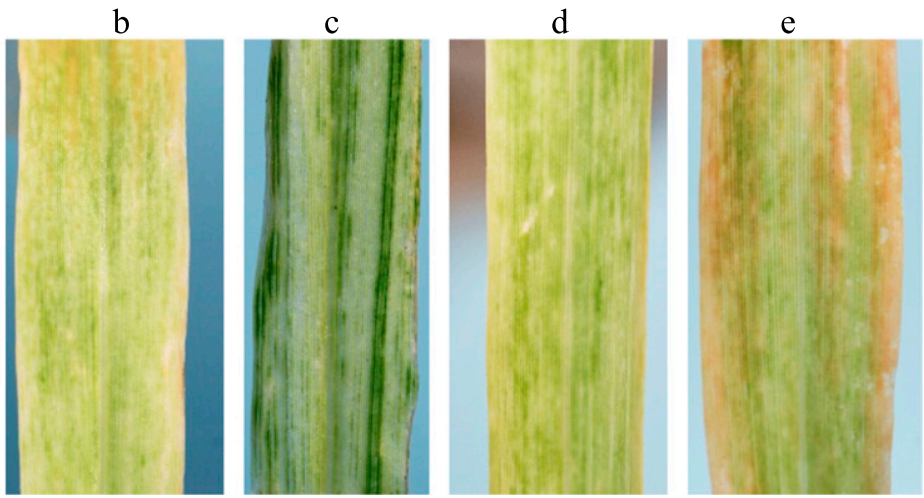

Fig. 6. Mutation of amino acids D35A, M98R, and D35A+M98R in coat protein (CP) of Satellite panicum mosaic virus (SPMV) SPMV-Type elicited enhanced disease synergism with Panicum mosaic virus (PMV) compared with wild-type SPMV-Type. A, Genomic organization of SPMV-Type (a), SPMV-KS (b), mutant SPMV-Type_D35A (c), mutant SPMV-Type_M98R (d), and SPMV-Type_D35A+M98R (e). Amino acid residues at positions 35 and 98 were mutated either singly or doubly in SPMV-Type as in SPMV-KS. B, Symptoms of proso millet leaves infected by coinoculation of PMV with either wild-type SPMV-KS, SPMV-Type, or SPMV-Type mutants at 14 days postinoculation. Note that infection of proso millet plants by PMV+SPMV-Type_D35A elicited moderate synergism, PMV+SPMV-Type_M98R induced moderate to severe disease synergism, and PMV+SPMV-Type_D35A+M98R elicited severe disease synergism. 
with a), suggesting that alanine residues at amino acid positions 18 and 59 were dispensable for augmented synergistic interaction with PMV. In contrast, mutant SPMV-KS_AAAM induced moderate symptoms similar to those by SPMV-Type
(Fig. 5B, column e compared with a and b), indicating that arginine at amino acid position 98 was crucial for enhanced synergistic interaction with PMV. Proso millet infected by a double mutant, SPMV-KS_ADSR, elicited moderate leaf
A

SPMV-KS
SPMV-Type
SPMV-KS
SPMV-Type
SPMV-KS
SPMV-Type

18

35

59

MAPKRSRRSNRRAGSRAAATSLVYDTCYVTLTERATTSFQRQSFPTLKGMGDRAFQVVAF 60 MAPKRSRRSNRRAGSRATATSLVYDTCYVTLTERDTTSFQRQSFPTLKGMGDRAFQVVSF 60 98

TIQGVSAAPLMYNARLYNPGDTDSVHATGVQLMGTVPRTVRLTPRVGQNNWFFGNTEEAE 120 TIQGVSAAPLMYNARLYNPGDTDSVHATGVQLMGTVPMTVRLTPRVGQNNWFFGNTEEAE 120

B

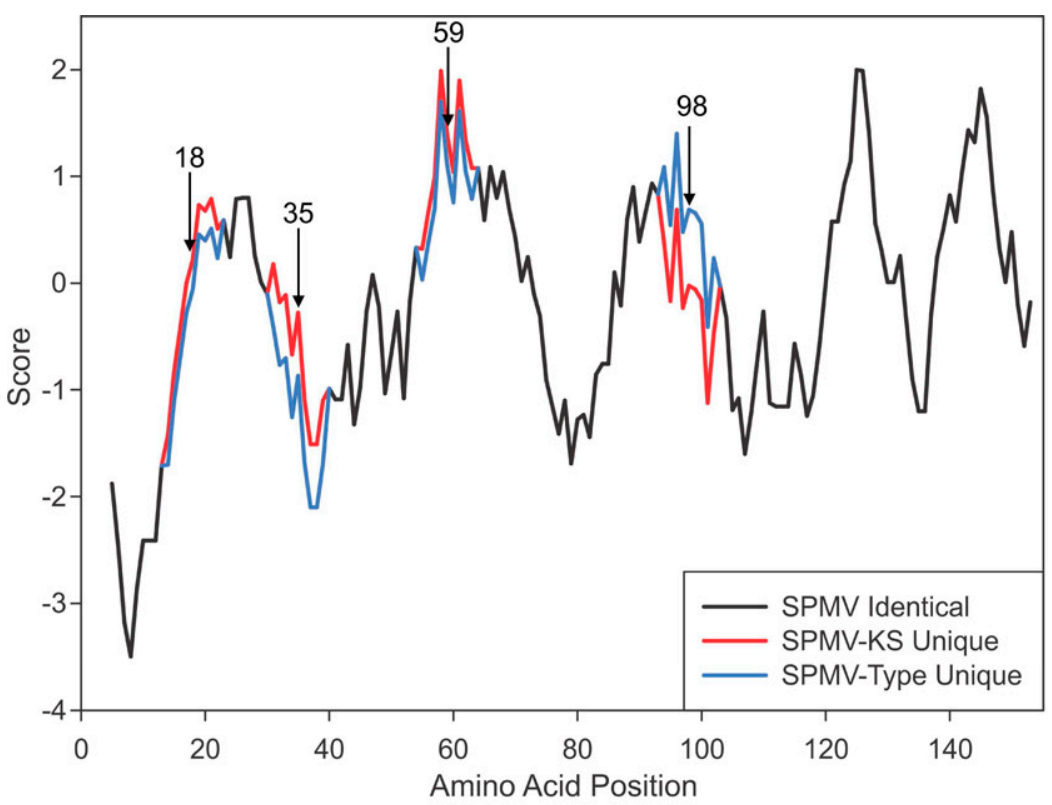

C

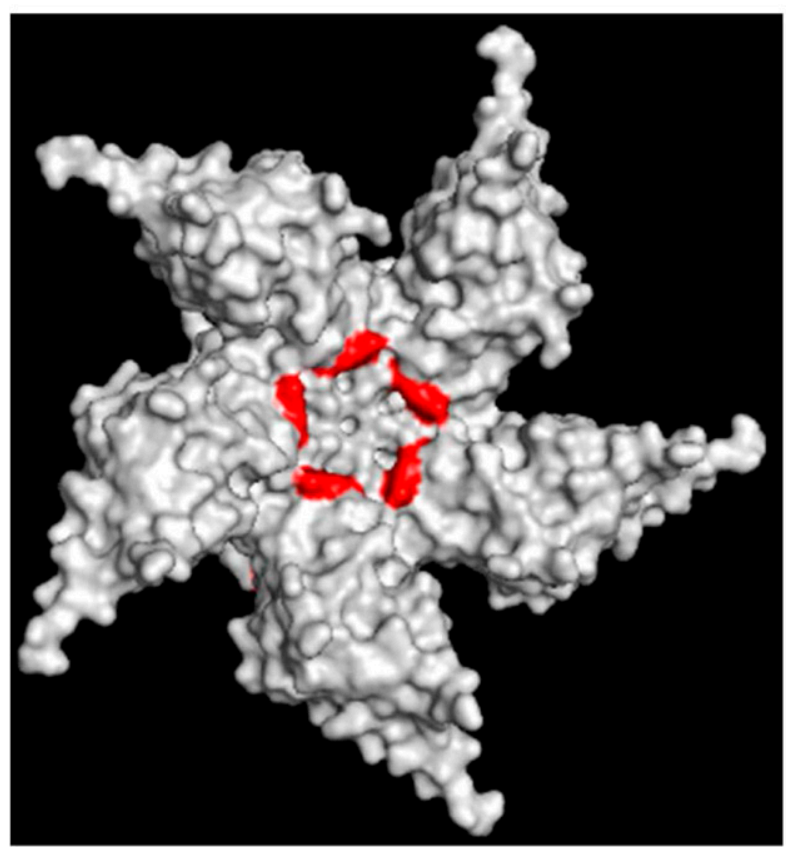

SPMV-KS CP

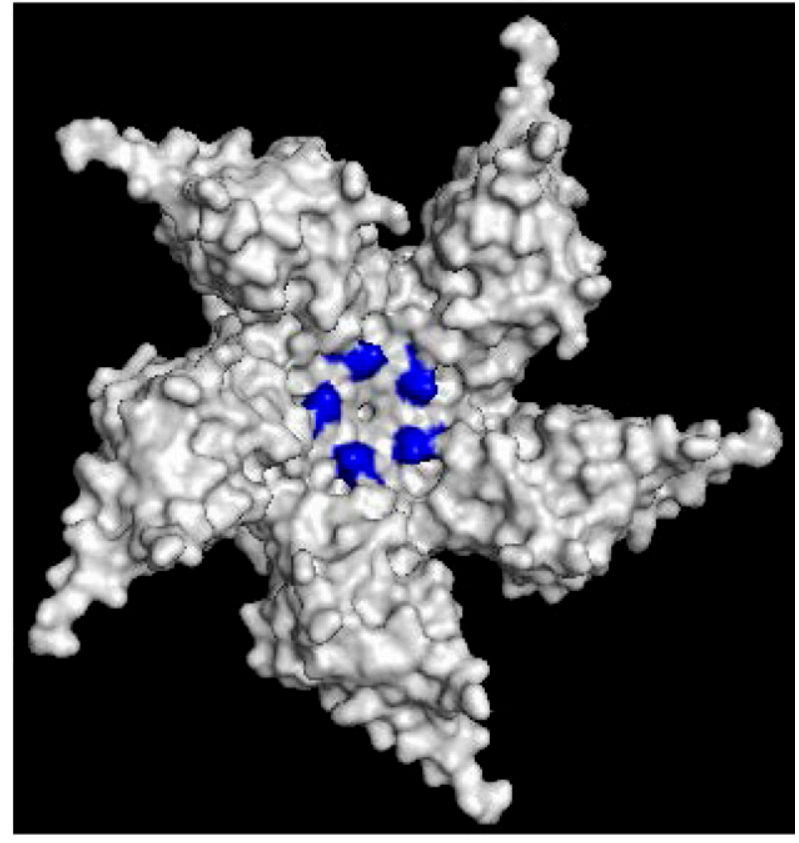

SPMV-Type CP 
chlorosis (Fig. 5B, column $\mathrm{f}$ ) and moderate stunting of plants (data not shown). These data suggest that alanine at amino acid position 35 was involved in efficient synergistic interactions with PMV, as mutation of alanine at amino acid position 59 has no effect on enhanced synergistic interaction with PMV. Triple mutants SPMV-KS_TDSR and SPMV-KS_ADSM elicited moderate symptoms on proso millet, similar to those of SPMVType (Fig. 5B, columns $g$ and $\mathrm{h}$ ), further confirming that alanine and arginine residues at amino acid positions 35 and 98 were crucial for enhanced synergistic interactions with PMV.

The role of CP amino acids at positions 35 and 98 in SPMV isolates in the differential synergistic interactions with PMV was further examined by mutating D35 and M98 either individually or together in SPMV-Type to obtain SPMVType_D35A, SPMV-Type_M98R, and SPMV-Type_D35A+ M98R, as in SPMV-KS (Fig. 6A). In-vitro transcripts of these mutants were coinoculated with PMV-NE onto proso millet seedlings at the two-leaf stage. Mutant SPMV-Type_D35A elicited moderately severe synergistic interaction on proso millet plants with large chlorotic streaks with a bleaching effect compared with medium-sized chlorotic streaks by wild-type SPMV-Type (Fig. 6B, columns c and a). Mutant SPMVType_M98R caused enhanced severe synergistic interaction on proso millet plants with severe chlorosis but a slightly milder synergism compared with wild-type SPMV-KS (Fig. 6B, column d compared with a and b). In contrast, SPMVType_D35A+M98R induced an augmented disease synergism with severe leaf chlorosis, stunting, leaf reddening, and death of most of the inoculated plants, similar to those elicited by SPMV-KS (Fig. 6B, column e compared with b). These data suggest that two amino acids at positions 35 and 98 in the $\mathrm{CP}$ ORFs of SPMV isolates were involved in the differential synergistic interactions with PMV isolates.

\section{Structure-function relationship analysis between CPs of SPMV isolates.}

The above data indicated that amino acids at positions 35 and 98 in the CP of SPMV isolates play a crucial role in differential synergistic interactions with PMV isolates. We next examined whether the functional differences found for amino acids 35 and 98 in the CP of SPMV isolates were reflected in predicted secondary structure and hydropathy of these two proteins. The protein structure was related to the D chain of the 1STM protein database of SPMV CP (Ban and McPherson 1995). The severe symptom-inducing SPMV-KS CP is identical in structure to 1STM and has A35 (neutral) and R98 (positively charged), whereas the moderate symptom-inducing SPMV-Type CP has D35 (negatively charged) and M98 (neutral) (Fig. 7A). ProtScale analysis (Gasteiger et al. 2005) of CP of SPMV isolates revealed subtle differences in the hydropathy of CPs at all four amino acid positions, with the greatest differences between SPMV-KS and SPMV-Type found around positions 35 and 98 (Fig. 7B). Additionally, differences in amino acids at positions 35 and 98 between SPMV-KS and SPMV-Type isolates also reflected changes in protein secondary structure in terms of length and repetitions of the $\beta$-strands and $\alpha$-helices (data not shown).
The I-TASSER program was used to predict and compare the three-dimensional structure of SPMV-Type to the known structure of SPMV-KS. Based on this predicted structure, amino acid changes of the two CPs were examined by PyMOL and Consurf server analyses (Ashkenazy et al. 2016). Subtle differences in the predicted structure of CP of SPMV-Type compared with that of SPMV-KS were observed (Fig. 7C). The Protein Data Bank structure of SPMV CP (1STM) indicated that amino acid residue 35 was on the external surface and residue 98 was present on the interior of the capsid. Residue 98 was part of the central cavity of the virion and occurred in the region that has been predicted to bind viral RNA (Ban and McPherson 1995). Predicted structures of SPMV-Type did not provide any obvious reason for how the change in A35D could significantly modify disease severity. However, in the solved structure of SPMV (Ban and McPherson 1995), A35 participates in main chain hydrogen bonding to G127. Substitution of A35D could potentially weaken this hydrogen-bonding to G127, since the $\beta$-carboxyl of D35 is bulkier and, possibly, either solvent-exposed, bound to a water molecule, or both. In the case of M98R (Fig. 7C), replacing a basic residue with a neutral one could subtly change the efficiency of encapsidating viral RNA, CP:RNA interactions, or both. Furthermore, R98 in the CP of SPMV-KS isolate can form a hydrogen bond with G64 (Ban and McPherson 1995). Potentially, M98 in the CP of SPMV-Type could change these interactions between the individual CP residues.

\section{DISCUSSION}

In this study, the genome of a Nebraska isolate of PMV, PMV-NE, was sequenced and infectious cDNA clones for PMV-NE and a Kansas isolate of SPMV, SPMV-KS, were constructed. Synergistic interactions between PMV (PMV-NE or PMV85) and SPMV (SPMV-KS or SPMV-Type) isolates were examined in proso millet. Infection of proso millet plants by either isolate of PMV in the presence of SPMV-KS elicited more severe disease symptoms compared with plants coinfected with either isolate of PMV and SPMV-Type. Exchange of fragments between SPMV-KS and SPMV-Type indicated that sequence differences in the CP of SPMV isolates were responsible for differential synergistic interactions with PMV isolates. The differential synergistic interactions of SPMV isolates with PMV were mapped to two amino acid residues in the SPMV CP at positions 35 and 98.

Stewart et al. (2015) reported widespread infection of PMV in switchgrass breeding plots in Nebraska between the 2012 and 2015 growing seasons. Increased incidence of PMV in switchgrass, together with the synergistic interactions between PMV and SPMV in proso and pearl millet plants with exacerbated symptoms (Scholthof 1999), warranted interaction studies between the isolates of PMV and SPMV. Though PMV was first reported from Kansas switchgrass breeding plots in 1953 (Sill and Pickett 1957), little attention was paid to PMV and SPMV infections in bioenergy-related switchgrass nurseries and plantations in Nebraska. The consensus sequence of PMV-NE,

Fig. 7. Amino acid sequence alignment, hydropathy plots, and predicted internal surface structures of Satellite panicum mosaic virus (SPMV) coat proteins (CPs) eliciting severe (SPMV-KS) and moderate (SPMV-Type) disease synergism with Panicum mosaic virus (PMV). A, Sequence alignment of CP amino acids of SPMV-KS and SPMV-Type. The location of four differing amino acids (in red font) between the CPs of SPMV-KS and SPMV-Type are indicated (starting from Met 1). B, Hydropathy prediction of CPs of SPMV-KS and SPMV-Type by ProtScale showing changes in the secondary structure of CPs at amino acid positions 18, 35, 59, and 98 (indicated with arrows). Note the subtle changes in predicted hydropathy score at amino acid positions 35 and 95 compared with amino acids at positions 18 and 59 between the CPs of SPMV isolates. C, Comparison of published structure of SPMV-KS (1STM1) (Ban and McPherson 1995 ) to predicted structure of SPMV-Type CP. Amino acid residue 98, which differs between SPMV-KS (in red) and SPMV-Type (in blue) isolates, is present on the interior of the capsid protein in the region that could be stabilized by binding to viral RNA. M98 (highlighted in blue) in SPMV-Type could potentially interfere with virion formation, CP-RNA interactions, or both and thereby affect synergistic interactions with PMV. 
obtained through high-throughput RNA-Seq from field-grown switchgrass, revealed that PMV-NE possesses 97\% sequence homology with isolate PMV85. However, SPMV sequences were not detected in these same RNA-Seq datasets, most likely due to technical difficulties.

Exacerbation of symptom severity of a helper virus in the presence of its molecular parasites (satellite viruses) is an intriguing phenomenon compared with traditional synergistic interactions between two independently replicating viruses (Dodds 1999; Rodríguez-Alvarado et al. 1994; Scholthof 1999; Syller 2012). Synergistic interactions between PMV85 and SPMV-Type were extensively studied with the exacerbated disease phenotype of PMV on millets compared with infections by PMV alone (Qi and Scholthof 2008, 2001a; Scholthof 1999). However, synergistic interactions between isolates of PMV and SPMV are not known. In this study, differential synergistic interactions between isolates of PMV and SPMV were observed. Coinfection of proso millet plants by PMV-NE and SPMV-KS elicited severe chlorosis, stunting, and death of plants, while coinfections by PMV85 and SPMV-Type induced chlorosis and long chlorotic streaks with moderate stunting of plants. These results indicated that either PMV-NE, SPMV-KS, or both possess determinants for enhanced synergistic interactions. Reciprocal experiments revealed that SPMV-KS but not PMV-NE was responsible for enhanced synergistic interactions in proso millet. Coinfection of proso millet plants by SPMV-KS and PMV-NE or PMV85 isolate elicited exacerbated disease symptoms and conclusively demonstrated that SPMV-KS was responsible for enhanced synergistic interactions. The genomic RNA copies and CP of both isolates of PMV increased significantly in proso millet plants coinfected with either isolate of SPMV. However, the genomic RNA copies and CP of SPMVKS accumulated persistently and at significantly higher levels, respectively, compared with those of SPMV-Type in proso millet plants coinfected with either isolate of PMV. These data suggest that increased accumulation of SPMV-KS CP most likely is responsible for enhanced synergistic interactions with PMV isolates.

Sequence analyses revealed that SPMV-KS and SPMV-Type isolates differed by $2 \mathrm{nts}$ in the $5^{\prime}$-UTR, by three synonymous and four nonsynonymous changes in the CP ORF, and by 3-nt changes in the $3^{\prime}$-UTR. Exchange of sequences between SPMV-KS and SPMV-Type revealed that SPMV hybrids comprising a SPMV-KS fragment between nts 63 and 457 (part of $5^{\prime}$-UTR and the N-terminal region of $\mathrm{CP}$ ) but not with a fragment between nt 457 and the $3^{\prime}$ end, elicited enhanced symptoms compared with SPMV-Type. These data revealed that four differential amino acids located in the CP ORFs between SPMV-KS and SPMV-Type were responsible for the differential synergistic interactions with PMV isolates. Previously, it has been reported that SPMV-Type CP was responsible for synergistic interactions with PMV85 (Omarov et al. 2005; Qi and Scholthof 2008; Qiu and Scholthof 2001a). Our data suggest that a few amino acid differences in the $\mathrm{CP}$ of SPMV can cause differential synergistic interactions with PMV.

Fine mapping of the four amino acids that were different between the CPs of SPMV-KS and SPMV-Type indicated that A18 and A59 did not have a significant role in differential synergistic interactions with the helper virus. However, A35 and R98 played critical roles in causing these differential synergistic interactions with PMV. Multiple functions have been reported for the CP of SPMV in systemic coinfection of millet plants with PMV (Omarov et al. 2005; Qi and Scholthof 2008; Qiu and Scholthof 2001a and b). Previous functional analyses of SPMV-Type CP revealed that the N-ARM motif was required for RNA accumulation, encapsidation, and RNAbinding activity, while the $\mathrm{C}$-terminal region was required for efficient RNA accumulation (Qi and Scholthof 2008). In-vitro RNA binding studies of SPMV CP deletion mutants revealed that the N-ARM and N-terminal regions were required for CP: RNA interactions, while progressive deletions toward the C-terminal region affected the efficiency of CP:RNA interactions (Qi and Scholthof 2008). It is possible that SPMV-KS mutants A35D and R98M might have affected the accumulation of CP, the CP:RNA interactions, and the efficiency of virion formation, which might have been responsible for reduced synergistic interactions compared with wild-type SPMV-KS. It is possible that amino acids at positions 35 and 98 in the CP of SPMV isolates might have differentially affected the efficiency of $\mathrm{CP}$ accumulation. This possibility, together with the persistent accumulation of SPMV-KS genomic RNA compared with that of SPMV-Type in proso millet plants, might have contributed to the enhanced synergistic interactions with PMV.

The available crystal structure of SPMV CP (Ban and McPherson 1995; Makino et al. 2006) facilitated the examination of effects of A35D and R98M on the SPMV CP secondary structure, as these mutations differentially affected the synergistic interactions with PMV isolates. In SPMV-Type, CP amino acid D35 is predicted to be on the surface and solventexposed, which could facilitate or compromise interactions with other $\mathrm{CP}$ amino acid residues, resulting in the modulation of efficient virus-host or virus-virus interactions, or both. Similarly, the positively charged amino acid R98 is present on the internal surface of the SPMV-KS CP and could potentially facilitate efficient interactions with negatively charged genomic RNAs of PMV, SPMV, or both, whereas neutral M98 in SPMV-Type might have affected these interactions. A few amino acid changes in viral proteins that can break resistance and symptom modulation have been reported (Chowda-Reddy et al. 2011; Karasawa et al. 1999). In contrast, mutations introduced in the CP of SPMV, a satellite virus, caused differential synergistic interactions with PMV. Potential differences in the accumulation of $\mathrm{CP}$ and genomic RNA copies between the isolates of SPMV, due to two differential amino acids at positions 35 and 98, are probable reasons for the observed differential disease synergism phenotypes. The mechanisms elucidating the role of the two differing amino acids between the CPs of SPMV isolates in differential synergistic interactions with PMV isolates are beyond the scope of the current investigation and will form the basis for future research.

\section{MATERIALS AND METHODS}

\section{PMV and SMPV isolates.}

Switchgrass cv. Summer rhizomes and flag leaves collected across three years, from 2010 to 2012, from the ENREC were used in this study as well as in other studies (Palmer et al. 2015, 2017). Total RNA extracted from these samples was used for high-throughput RNA-Seq at the University of Nebraska Medical Center (Omaha, NE, U.S.A.). RNA-Seq reads (100 bp) not aligning to the switchgrass genome were aligned to the PMV genome sequence available in GenBank (accession number U55002) to obtain a consensus genome sequence of the PMV Nebraska population (GenBank accession number MH885652). Infectious cDNA clones of PMV85 (Turina et al. 1998) and SPMV-Type (Qiu and Scholthof 2000; Scholthof 1999) were obtained from K.-B. Scholthof, Texas A\&M University (College Station, TX, U.S.A.).

\section{Construction of infectious cDNA clones of PMV-NE and SPMV-KS isolates and chimeras between SPMV-KS and SPMV-Type isolates.}

Double-stranded cDNAs for the genomic RNAs of PMV-NE and SPMV-KS (GenBank accession number M17182) were 
synthesized at GenScript USA Inc., followed by ligation into pUC57-Mini at the EcoRV restriction site. Unique SpeI (nt 63) and $M s c \mathrm{I}$ (nt 457) restriction sites located in the genomes of SPMV-KS and SPMV-Type isolates were used to exchange the genomic RNA fragments between SPMV isolates. Mutation of $\mathrm{CP}$ amino acid codons at positions 18, 35, 59, and 98 in SPMV$\mathrm{KS}$, and of amino acid codons 35 and 98 in SPMV-Type were performed by overlap extension PCR. In-vitro RNA transcripts were generated in a $40-\mu \mathrm{l}$ reaction from $1.0 \mu \mathrm{g}$ of linearized plasmid DNAs and were inoculated onto 12 to 15 proso millet seedlings at the two-leaf stage, as described by Tatineni et al. (2011). For coinoculations, $80 \mu \mathrm{l}$ of in-vitro RNA transcripts containing $40 \mu \mathrm{l}$ each for PMV and SPMV was inoculated onto proso millet seedlings at the two-leaf stage. Symptom expression of PMV or PMV+SPMV on proso millet plants was observed at 14 and $21 \mathrm{dpi}$. Two independent clones for each construct were used for phenotypic assays, and each experiment was repeated at least three times. Plasmid cDNAs and agarose gel-isolated RT-PCR products of mutant viruses from total RNA isolated from virus-infected proso millet plants were sequenced for the presence of introduced mutations.

\section{RT-PCR.}

Total RNA was extracted from symptomatic leaves of proso millet plants by TriPure isolation reagent (Roche, Indianapolis, IN, U.S.A.) at 14 and $21 \mathrm{dpi}$. One microgram of total RNA was used for reverse transcription (New England Biolabs, Ipswich, MA, U.S.A.) with random hexamer primers, followed by RTqPCR with the SsoAdvanced SYBR green supermix (Bio-Rad, Hercules, CA, U.S.A.) in the Bio-Rad CFX Connect real-time PCR system. RT-qPCR was performed on cDNA from 14- and 21-dpi samples, with four replicates per treatment combination, with oligonucleotides PMV-RT-F (5'-TGGCTGCAACCYA TACCTGA-3', corresponding to nts 5 to 25 in the PMV genome; $\mathrm{Y}=\mathrm{C}$ or $\mathrm{T})$ and PMV-RT-R (5'-CCTGTGGGAA GCGTGTAAGT-3', complementary to nts 122 to 102 in the PMV genome) for PMV and oligonucleotides SPMV-RT-F (5'-ATTCCAACGCTAGCAACGAG-3', corresponding to nts 7 to 27 in the SPMV genome) and SPMV-RT-R (5- GACGA TTAGATCGCCTGGAA-3', complementary to nts 131 to 111 in the SPMV genome) for SPMV. RT-qPCR was performed using the following conditions: $95^{\circ} \mathrm{C}$ for $30 \mathrm{~s}$, followed by 40 cycles of $95^{\circ} \mathrm{C}$ for $5 \mathrm{~s}, 60^{\circ} \mathrm{C}$ for $10 \mathrm{~s}$, followed by a melt curve analysis $\left(65\right.$ to $95^{\circ} \mathrm{C}$ at $0.2^{\circ} \mathrm{C}$ increments) to verify single PCR products. RT-qPCR reactions, without RNA template and reverse transcription, were included as negative controls. The relative expression of PMV and SPMV genomic RNA copies was calculated by using the cycle threshold $(\Delta \mathrm{Ct})$ method, using casein kinase 1-like protein 2 and 26S proteasome regulatory subunit S2 1A genes as internal controls for normalization (Donze-Reiner et al. 2017).

\section{Analysis of total proteins for accumulation of CPs of PMV and SPMV.}

Total proteins were extracted from $50 \mathrm{mg}$ of PMV- or PMV+ SPMV-infected proso millet leaves at $14 \mathrm{dpi}$ in $250 \mu \mathrm{l}$ of protein extraction buffer $(50 \mathrm{mM}$ Tris acetate, $\mathrm{pH} 7.4,10 \mathrm{mM}$ potassium acetate, $1 \mathrm{mM}$ EDTA, $5 \mathrm{mM}$ dithiothreitol containing one complete mini protease inhibitor cocktail tablet [Roche] per $10 \mathrm{ml}$ ), as described by Tatineni et al. (2011). The macerate was clarified at $4^{\circ} \mathrm{C}$ for $10 \mathrm{~min}$ at $15,000 \times \mathrm{g}$. Five micrograms of total proteins per sample were loaded onto 4 to $12 \%$ SDS-PAGE gel, followed by staining with Coomassie Brilliant Blue R-250.

\section{Secondary structure analysis of CP mutants of SPMV.}

HHpred (Biegert et al. 2006) and PHYRE (Kelley and Sternberg 2009) was used for SPMV CP secondary structure prediction. The published crystal structure for SPMV CP is 1STM, except for the N-terminal 16 amino acids (Ban and McPherson 1995). The I-TASSER program (Roy et al. 2010) was used to model the full-length SPMV CP, using 1STM as the template. PyMOL software was used to construct the predicted secondary structures of SPMV CP mutants. Hydropathy of SPMV CP sequences was predicted using Kyte and Doolittle options in ProtScale (Gasteiger et al. 2005). The postscript and verbose files were used to generate the changes in hydropathy scale at their respective amino acid positions.

\section{ACKNOWLEDGMENTS}

We thank K.-B. Scholthof, Texas A\&M University for providing pPMV85 and pSPMV-Type used in this investigation.

\section{LITERATURE CITED}

Ashkenazy, H., Abadi, S., Martz, E., Chay, O., Mayrose, I., Pupko, T., Ben-Ta, N. 2016 ConSurf 2016: An improved methodology to estimate and visualize evolutionary conservation in macromolecules. Nucleic Acids Res. 44:W344-W350.

Ban, N., and McPherson, A. 1995. The structure of satellite panicum mosaic virus at 1.9 A resolution. Nat. Struct. Biol. 2:882-890.

Batten, J. S., and Scholthof, K.-B. G. 2004. St Augustine decline. Pages 800-802 in: Viruses and virus diseases of Poaceae (Gramineae). H. Lapierre and P. Signore, eds. INRA, Paris.

Batten, J. S., Turina, M., and Scholthof, K.-B. G. 2006. Panicovirus accumulation is governed by two membrane-associated proteins with a newly identified conserved motif that contributes to pathogenicity. Virol. J. 3:12-23.

Biegert, A., Mayer, C., Remmert, M., Söding, J., and Lupas, A. N. 2006. The MPI bioinformatics toolkit for protein sequence analysis. Nucleic Acids Res. 34:W335-W339.

Chowda-Reddy, R. V., Sun, H., Chen, H., Poysa, V., Ling, H., Gijzen, M., and Wang, A. 2011. Mutations in the P3 protein of Soybean mosaic virus G2 isolates determine virulence on Rsv4-genotype soybean. Mol. PlantMicrobe Interact. 24:37-43.

Dodds, J. A. 1999. Satellite tobacco mosaic virus. Curr. Top. Microbiol. Immunol. 239:145-157.

Donze-Reiner, T., Palmer, N. A., Scully, E. D., Prochaska, T. J., Koch, K. G., Heng-Moss, T., Bradshaw, J. D., Twigg, P., Amundsen, K., Sattler, S. E., and Sarath, G. 2017. Transcriptional analysis of defense mechanisms in upland tetraploid switchgrass to greenbugs. BMC Plant Biol. 17:46.

Gasteiger, E., Hoogland, C., Gattiker, A., Duvaud, S., Wilkins, M. R., and Appel, R. D. 2005. Bairoch A.; Protein Identification and Analysis Tools on the ExPASy Server. Pages 571-607 in: The Proteomics Protocols Handbook. J. M. Walker, ed. Humana Press, Totowa, NJ, U.S.A.

Karasawa, A., Okada, I., Akashi, K., Chida, Y., Hase, S., Nakazawa-Nasu, Y., Ito, A., and Ehara, Y. 1999. One amino acid change in cucumber mosaic virus RNA polymerase determines virulent/avirulent phenotypes on cowpea. Phytopathology 89:1186-1192.

Kelley, L. A., and Sternberg, M. J. E. 2009. Protein structure prediction on the Web: A case study using the Phyre server. Nat. Protoc. 4:363-371.

Makino, D. L., Day, J., Larson, S. B., and McPherson, A. 2006. Investigation of RNA structure in satellite panicum mosaic virus. Virology 351:420-431.

Mandadi, K. K., Pyle, J. D., and Scholthof, K.-B. G. 2015. Characterization of SCL33 splicing patterns during diverse virus infections in Brachypodium distachyon. Plant Signal. Behav. 10:e1042641.

Mandadi, K. K., and Scholthof, K.-B. G. 2012. Characterization of a viral synergism in the monocot Brachypodium distachyon reveals distinctly altered host molecular processes associated with disease. Plant Physiol. 160:1432-1452.

Mandadi, K. K., and Scholthof, K.-B. G. 2015. Genome-wide analysis of alternative splicing landscapes modulated during plant-virus interactions in Brachypodium distachyon. Plant Cell 27:71-85.

Mascia, T., and Gallitelli, D. 2016. Synergies and antagonisms in virus interactions. Plant Sci. 252:176-192.

Masuta, C., Zuidema, D., Hunter, B. G., Heaton, L. A., Sopher, D. S., and Jackson, A. O. 1987. Analysis of the genome of satellite panicum mosaic virus. Virology 159:329-338.

Omarov, R. T., Qi, D., and Scholthof, K.-B. G. 2005. The capsid protein of satellite panicum mosaic virus contributes to systemic invasion and interacts with its helper virus. J. Virol. 79:9756-9764. 
Palmer, N. A., Donze-Reiner, T., Horvath, D., Heng-Moss, T., Waters, B., Tobias, C., and Sarath, G. 2015. Switchgrass (Panicum virgatum L) flag leaf transcriptomes reveal molecular signatures of leaf development, senescence, and mineral dynamics. Funct. Integr. Genomics 15:1-16.

Palmer, N. A., Saathoff, A. J., Scully, E. D., Tobias, C. M., Twigg, P., Madhavan, S., Schmer, M., Cahoon, R., Sattler, S. E., Edmé, S. J., Mitchell, R. B., and Sarath, G. 2017. Seasonal below-ground metabolism in switchgrass. Plant J. 92:1059-1075.

Pyle, J. D. 2015. Molecular pathogenesis of viral and subviral agents in model and crop grasses. Page 152. Master's thesis, Texas A\&M University, College Station, TX, U.S.A..

Pyle, J. D., Monis, J., and Scholthof, K.-B. G. 2017. Complete nucleotide sequences and virion particle association of two satellite RNAs of panicum mosaic virus. Virus Res. 240:87-93.

Qi, D., and Scholthof, K.-B. G. 2008. Multiple activities associated with the capsid protein of satellite panicum mosaic virus are controlled separately by the $\mathrm{N}$ - and C-terminal regions. Mol. Plant-Microbe Interact. 21:613-621.

Qiu, W., and Scholthof, K.-B. G. 2000. In vitro- and in vivo-generated defective RNAs of satellite panicum mosaic virus define cis-acting RNA elements required for replication and movement. J. Virol. 74:2247-2254.

Qiu, W., and Scholthof, K.-B. G. 2001a. Genetic identification of multiple biological roles associated with the capsid protein of satellite panicum mosaic virus. Mol. Plant-Microbe Interact. 14:21-30.

Qiu, W., and Scholthof, K.-B. G. 2001b. Defective interfering RNAs of a satellite virus. J. Virol. 75:5429-5432.

Qiu, W., and Scholthof, K.-B. G. 2004. Satellite panicum mosaic virus capsid protein elicits symptoms on a nonhost plant and interferes with a suppressor of virus-induced gene silencing. Mol. Plant-Microbe Interact. 17:263-271.

Rodríguez-Alvarado, G., Kurath, G., and Dodds, J. A. 1994. Symptom modification by satellite tobacco mosaic virus in pepper types and cultivars infected with helper tobamoviruses. Phytopathology 84: 617-621.

Roy, A., Kucukural, A., and Zhang, Y. 2010. I-TASSER: A unified platform for automated protein structure and function prediction. Nat. Protoc. 5: 725-738.

Scholthof, K.-B. G. 1999. A synergism induced by satellite panicum mosaic virus. Mol. Plant-Microbe Interact. 12:163-166.

Scholthof, K.-B. G., Jones, R. W., and Jackson, A. O. 1999. Biology and structure of plant satellite viruses activated by icosahedral helper viruses. Pages in 123-143. Satellites and defective Viral RNAs. P. K. Vogt and A. O. Jackson, ed. Springer, Berlin.

Sill, W. H., and Pickett, R. C. 1957. A new disease of switchgrass, panicum virgatum. Plant Dis. Rep. 41:241-249.

Stewart, C. L., Pyle, J. D., Jochum, C. C., Vogel, K. P., Yuen, G. Y., and Scholthof, K.-B. G. 2015. Multi-year pathogen survey of biofuel switchgrass breeding plots reveals high prevalence of infections by Panicum mosaic virus and its satellite virus. Phytopathology 105: 1146-1154.

Syller, J. 2012. Facilitative and antagonistic interactions between plant viruses in mixed infections. Mol. Plant Pathol. 13:204-216.

Tatineni, S., McMechan, A. J., Hein, G. L., and French, R. 2011. Efficient and stable expression of GFP through Wheat streak mosaic virus-based vectors in cereal hosts using a range of cleavage sites: Formation of dense fluorescent aggregates for sensitive virus tracking. Virology 410: 268-281.

Turina, M., Desvoyes, B., and Scholthof, K.-B. G. 2000. A gene cluster encoded by panicum mosaic virus is associated with virus movement. Virology 266:120-128.

Turina, M., Maruoka, M., Monis, J., Jackson, A. O., and Scholthof, K.-B. G. 1998. Nucleotide sequence and infectivity of a full-length cDNA clone of panicum mosaic virus. Virology 241:141-155. 